\title{
Resección de metástasis pulmonares en cánceres ginecológicos: Presentación de un caso y análisis de la literatura
}

\author{
José M. Clavero $\mathbf{R}^{1}$, Mauricio Cuello $F^{2}$, \\ Adriana Castiblanco $\mathrm{G}^{3}$, Rodrigo A paricio $\mathbf{R}^{1}$. \\ Pulmonary resection of lung \\ metastases of an uterine \\ leiomyosarcoma. Report of one case
}

We report a 45 years-old woman operated in 2003 for a stage IV uterine leiomyosarcoma with lung metastases. Pulmonary metastases were surgically excised in 2003, 2005 and finally in July, 2006. Since then, the patient is in good conditions. There is evidence that resection of pulmonary metastases of gynecologic cancers, when the primary tumor is controlled, significantly prolongs survival (Rev Méd Chile 2007; 135: 1171-77).

(Key w ords: Leiomyosarcoma; Lung neoplasms; Neoplasm metastasis; Uterine neoplasms)

Recibido el 3 de mayo, 2006. Aceptado el 6 de noviembre, 2006.

${ }^{1}$ Sección Cirugía de Tórax, División de Cirugía. ${ }^{2}$ Departamento de Obstetricia y Ginecología. ${ }^{3}$ Departamento de Anatomía Patológica, Escuela de Medicina, Pontificia Universidad Católica de Chile. Santiago de Chile.

L as primeras resecciones de metástasis pulmonares fueron efectuadas por JW Weinlechner y RU Kronlein en 1882 y 1883, respectivamente ${ }^{1,2}$. La primera lobectomía por metástasis pulmonares fue realizada por CE Churchill en 1939 en un paciente con cáncer renal quien sobrevivió 23 años, falleciendo finalmente por enfermedad cardiovascular ${ }^{3}$.

Desde estos inicios, las resecciones de metástasis pulmonares sólo se efectuaron en forma esporádica en grupos muy seleccionados de pa-

Correspondencia a: Dr. José Miguel Clavero R. Sección Cirugía de Tórax, División de Cirugía. Pontificia Universidad Católica de Chile. Marcoleta 351 interior, $2^{\circ}$ piso. Santiago de Chile. E mail: jclavero@med.puc.cl cientes. Estudios posteriores demostraron un claro beneficio, en términos de sobrevida, en pacientes seleccionados. Actualmente la resección de metástasis pulmonares es un tratamiento aceptado en diferentes tumores primarios ${ }^{4-6}$.

A diferencia de otros tumores sólidos existe menos evidencia del rol de la resección de metástasis pulmonares en pacientes con tumores de origen ginecológico. Presentamos el caso de una paciente de 31 años con un leiomiosarcoma uterino sometida a resección de metástasis pulmonares.

\section{CASO CLÍNICO}

Paciente de 45 años, con antecedentes de hipertensión arterial crónica en tratamiento con atenolol (50 
$\mathrm{mg} /$ día) e hidroclorotiazida $12,5 \mathrm{mg}$ + triamterene $25 \mathrm{mg}$ tres veces por semana. Multípara de 2 hijos, usuaria de dispositivo intrauterino ( $\mathrm{T}$ de cobre, por 7 años). Consultó por hipermenorrea y dismenorrea de larga data, de intensidad progresiva y resistente al tratamiento médico (antiinflamatorios no esteroidales y retiro del dispositivo intrauterino). $\mathrm{Al}$ examen físico presentaba un útero aumentado de tamaño ( \pm 15 semanas), de forma nodular, con anexos normales. Se solicitó ultrasonografía transvaginal que confirmó la presencia de miomas múltiples, el mayor en el fondo uterino de $4 \mathrm{~cm}$ de diámetro. El hemograma demostró anemia ferropriva significativa (Hto. 26\%).

Dado el tamaño de los miomas y síntomas asociados (hipermenorrea, anemia severa secundaria, dismenorrea y dispareunia intensa), se decidió realizar un tratamiento quirúrgico. El 21 de julio de 2003 se realizó una histerectomía total con conservación de anexos. Sólo se evidenció la presencia de miomas múltiples, sin otros hallazgos significativos. Evolucionó satisfactoriamente, dándose de alta en buenas condiciones con suplemento oral de fierro.

El estudio anatomopatológico reveló un leiomiosarcoma uterino de $8 \mathrm{~cm}$ de diámetro mayor, con alto índice mitótico (46 a 62 mitosis por 10 campos de aumento mayor) y elevado número de atipias (Figura 1). El estudio inmunohistoquímico fue positivo para actina y desmina en las células tumorales. Con estos hallazgos fue derivada a nuestro Centro de Cáncer, siendo evaluada por ginecología oncológica, solicitándose estudio de diseminación. En la tomografía computada de tórax se demostró un nódulo pulmonar en el lóbulo superior izquierdo, sin evidencias de otras localizaciones secundarias (Figura 2). Se discutió en Comité Oncológico y se decidió resecar el nódulo. El 23 de septiembre de 2003 se realizó una toracotomía izquierda y resección de dos nódulos pulmonares. El estudio anatomopatológico confirmó la presencia de metástasis pulmonares de leiomiosarcoma uterino en ambos (Figura 2). Se decidió posteriormente mantener en seguimiento estricto. Se mantuvo en buenas condiciones, realizando vida normal y reintegrándose a su trabajo. En control de febrero de 2004 se evidenció nuevo nódulo pequeño en lóbulo inferior izquierdo. Se discutió nuevamente el caso en Comité Oncológico, decidiéndose seguimiento estricto y resección quirúrgica en caso de progresión. Permaneció en buenas condiciones hasta fines de 2004, en que se constató crecimiento del nódulo pulmonar, sin evidencia de otras lesiones secundarias (Figura 3).

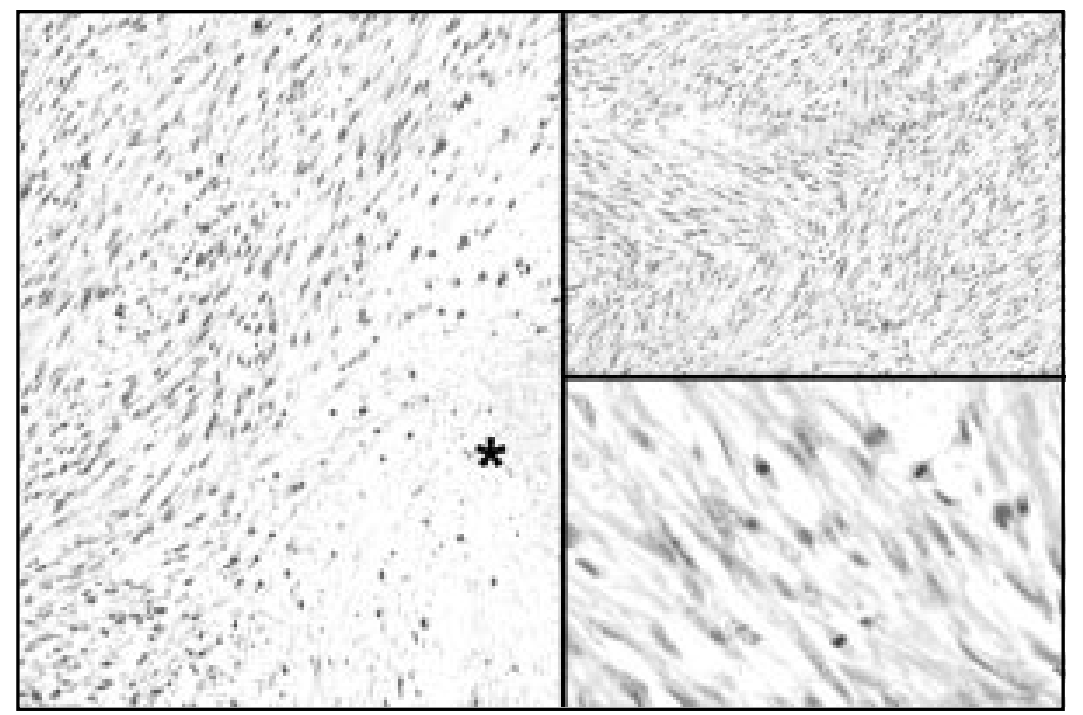

Figura 1. Aspecto microscópico del tumor primario, un leiomiosarcoma intramural uterino. Nótese que el tumor presenta intenso pleomorfismo celular, necrosis de coagulación $(*)$, alto recuento mitótico y atipias (hematoxilina-eosina x100, x200, x400). 


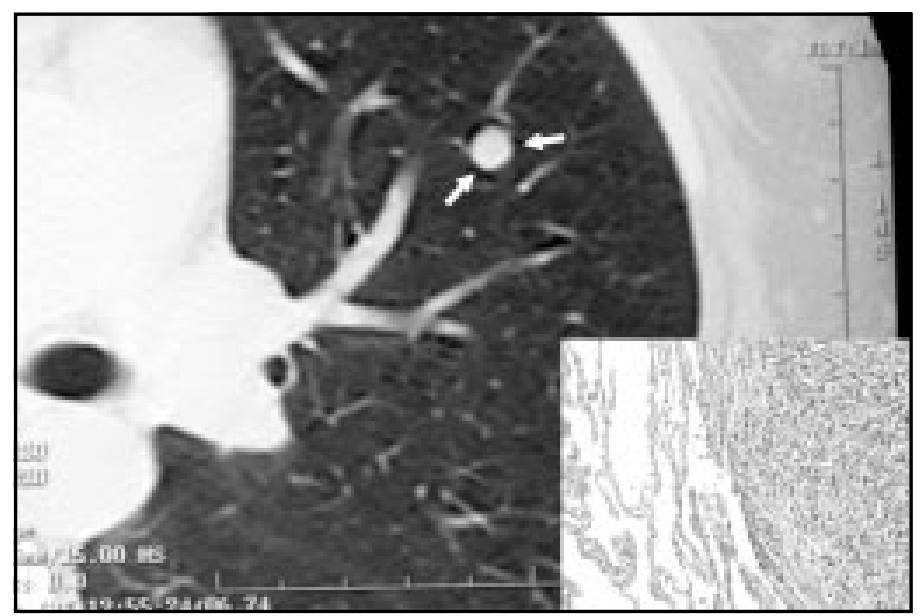

Figura 2. Tomografía axial computada de tórax que muestra nódulo pulmonar de $9 \mathrm{~mm}$ en el segmento anterior del lóbulo superior izquierdo (indicado por las flechas blancas). En el recuadro se aprecia el aspecto histológico del nódulo, similar al del tumor primario. El nódulo se encuentra rodeado de parénquima pulmonar sano (hematoxilinaeosina, $\mathrm{x} 200$ ).

Figura 3. Tomografía axial computada de tórax que muestra nuevo nódulo pulmonar de $9 \mathrm{~mm}$ (indicado por flechas blancas) detectado a 6 meses de primera resección. Sobre dicho nódulo se aprecia cicatriz de resección anterior. Por crecimiento durante seguimiento se reopera. En el recuadro se aprecia su aspecto histológico (hematoxilina-eosina, $\mathrm{x} 400$ ).

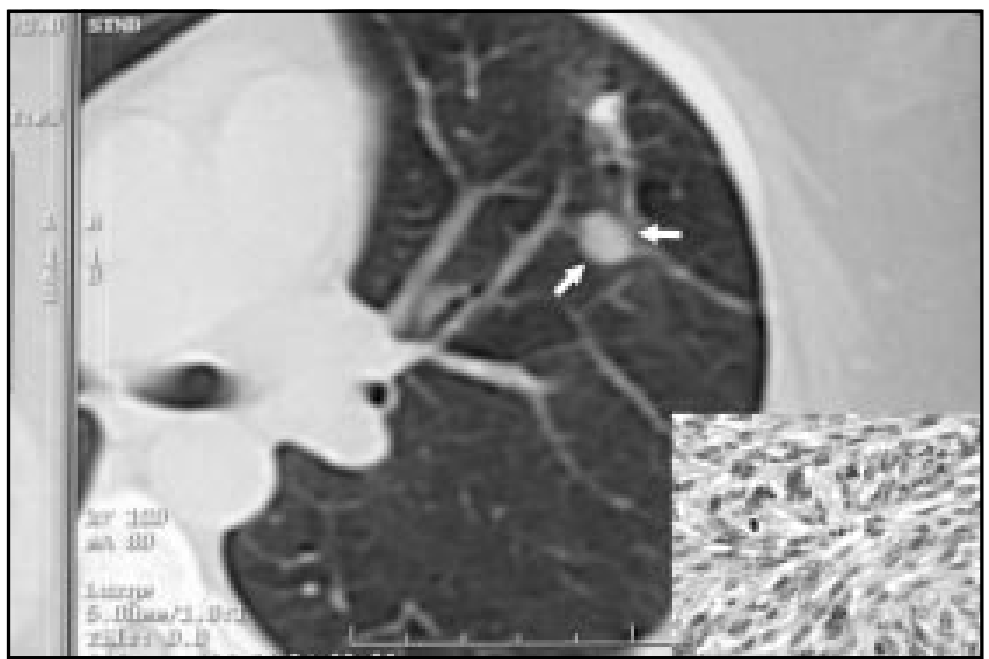

Por lo que en marzo de 2005 se realizó una retoracotomía izquierda y resección de metástasis. El estudio anatomopatológico confirmó la resección completa de dos metástasis pulmonares de leiomiosarcoma (Figura 3). La paciente se recuperó sin inconvenientes y se reincorporó a su trabajo manteniéndose en seguimiento estricto. A mediados de 2006 se pesquisaron 2 nuevos nódulos pulmonares en el lóbulo inferior izquierdo, sin evidencia de enfermedad metastásica en otras localizaciones. Se realizó una nueva resección de metástasis en julio de 2006, resecándose 2 nuevas metástasis. Desde esa fecha hasta el presente se ha mantenido en buenas condiciones generales completando 36 meses de seguimiento.

\section{DisCUSIÓN}

Las metástasis pulmonares representan la neoplasia pulmonar más frecuente. Se encuentran en cerca de $30 \%$ de los pacientes fallecidos por cáncer y en aproximadamente $20 \%$ de ellos el pulmón es el único órgano afectado ${ }^{5}$.

Los criterios generales para la resección de metástasis pulmonares han sido claramente establecidos: el tumor primario debe estar controlado o ser controlable, no debe existir evidencia de metástasis extrapulmonares y el paciente debe tener una adecuada reserva pulmonar que permita realizar una resección completa de todas las metástasis ${ }^{4-6}$. 
La sobrevida a cinco años de los pacientes sometidos a resección de metástasis pulmonares varía entre $20 \%$ y $40 \%$ en diferentes series ${ }^{6-17}$. Los factores pronósticos más importantes que han sido identificados son: el intervalo libre de enfermedad (tiempo transcurrido entre el tratamiento del tumor primario y la aparición de las metástasis pulmonares), el número de metástasis y la realización de una resección completa ${ }^{4,78}$. Otros factores pronósticos son el tamaño de las metástasis, el tiempo de duplicación tumoral (tiempo que necesita la metástasis para doblar su volumen), presencia de metástasis bilaterales y compromiso de los linfonodos mediastínicos $5,6-20$.

El tipo de cirugía depende del número y localización de las metástasis. Los principios quirúrgicos son la realización de una resección completa de todas las metástasis presentes al momento de la cirugía. La cirugía de elección es la resección en cuña de la metástasis, sin embargo en ocasiones es necesario realizar resecciones mayores como lobectomías o bilobectomías y muy ocasionalmente neumonectomías. La vía de abordaje es por toracotomía posterolateral uni 0 bilateral, toracotomía anterior o esternotomía media. El rol de la videotoracoscopia no está claramente establecido ${ }^{4,21}$. Con la palpación directa del pulmón durante una toracotomía es posible encontrar $12,5 \%$ más de metástasis pulmonares que las sospechadas por $\mathrm{TAC}^{22}$ y hasta $56 \%$ más que las encontradas por videotoracoscopia ${ }^{23}$. Por lo que en general se prefiere realizar una cirugía abierta para la resección de metástasis con intención curativa. La videotoracoscopia es preferida para confirmar la presencia de metástasis pulmonares en pacientes que no son candidatos a cirugía resectiva, para certificar el diagnóstico previo a la quimioterapia o para efectuar tratamiento paliativo ${ }^{4}$.

El cáncer se ha convertido en una de las principales causas de muerte en la población femenina. En relación a los tumores de origen ginecológico, se estima que 80.000 mujeres desarrollarán un cáncer de este tipo en Estados Unidos de Norteamérica durante el año 2006 y de ellas $36 \%$ morirá finalmente como consecuencia de su cáncer ${ }^{24}$.

En nuestro país el cáncer es la segunda causa de muerte, correspondiendo el año 2003 a 23,5\% del total de muertes. De las 38.190 mujeres que fallecie- ron ese año, 8.812 fallecieron por cáncer y de éstas $1.264(14,3 \%)$ por cánceres de origen ginecológico, incluyendo cuello uterino, cuerpo uterino, ovario, vulva, vagina y otros mal definidos ${ }^{25}$.

Las metástasis pulmonares en los tumores de origen ginecológico son frecuentes, con una incidencia de $2 \%$ a $28 \%{ }^{26-29}$. Pese a ser poco conocido, la resección de metástasis pulmonares de estos tumores ha demostrado prolongar la sobrevida en casos seleccionados. La primera resección de una metástasis pulmonar de un cáncer ginecológico fue realizada por Torek en 1930, en una paciente con un adenocarcinoma uterino ${ }^{30}$. Desde entonces se han realizado resecciones de metástasis pulmonares de diferentes cánceres ginecológicos con resultados similares a lo observado en otros tumores primarios $7,19,26-28,31-33$

En el cáncer cérvico uterino se describe una incidencia de metástasis pulmonares entre $2 \%$ y $9 \%{ }^{27,28}$. La incidencia es mayor en estadios avanzados y en tumores más indiferenciados, siendo frecuente la presencia de metástasis extrapulmonares ${ }^{28,34}$. En diferentes series de pacientes sometidas a resección de metástasis pulmonares, siguiendo los criterios de selección previamente mencionados, la sobrevida a 5 años ha sido de $24 \%$ a $53 \% 26,35,36$.

La incidencia de metástasis pulmonares en cáncer de endometrio es de $6 \%$, siendo la mayoría metacrónicas ${ }^{37}$. En los estudios de pacientes sometidas a resección de metástasis pulmonares el intervalo libre de enfermedad luego de la resección ha sido de 21 a 29 meses, la sobrevida media de 15,7 a 81 meses y la sobrevida a cinco años de $57,2 \%$ a $75,7 \% 31,32,36$.

Los sarcomas uterinos representan $2 \%$ a $5 \%$ de las neoplasias uterinas ${ }^{38}$, se originan de dos líneas tisulares: los leiomiosarcomas del músculo miometrial y los tumores mesodérmicos (Müllerianos) y los sarcomas estromales del epitelio endometrial. Los tipos histológicos más frecuentes son el carcinosarcoma (sarcoma mixto mesodérmico) que representa $50 \%$ de estos tumores, el leiomiosarcoma que representa $30 \%$ y el sarcoma endometrial estromal que representa 15\%. El pronóstico depende de la extensión de la enfermedad al momento del diagnóstico ${ }^{39}$. La sobrevida a cinco años es de $50 \%$ en tumores etapa I (tumores confinados al cuerpo uterino) y entre $0 \%$ y $20 \%$ en etapas más avanzadas ${ }^{39}$. El tratamiento 
de elección es la resección quirúrgica completa en los tumores limitados al útero. No existe evidencia sustancial de que la quimioterapia o la radioterapia adyuvante sean de beneficio en este tipo de sarcomas, aunque es efectuado en muchos centros por su alto riesgo de recurrencia ${ }^{40,41}$. No existe tampoco un tratamiento aceptado para los sarcomas uterinos metastáticos. Se han efectuado diferentes estudios fase II con diferentes drogas de quimioterapia con regulares resultados. En tumores mesodérmicos la ifosfamida ha tenido $32,2 \%$ de respuesta y $33 \%$ en sarcomas estromales endometriales, pero sólo 17,2\% de respuesta parcial en leiomiosarcomas ${ }^{42-44}$. La terapia combinada se ha utilizado en tumores mesodérmicos, sin que se haya demostrado beneficios con el uso de cisplatino-ifosfamida sobre ifosfamida sola ${ }^{45}$.

La incidencia de metástasis pulmonares en sarcomas uterinos es de $4 \%$ en general y de $7 \%$ a $28 \%$ en los sarcomas estromales endometria$\operatorname{les}^{29,46}$. No existen estudios randomizados entre quimioterapia y resección de metástasis pulmonares, y los estudios de quimioterapia incluyen pacientes con metástasis pulmonares y enfermedad metastásica en otras localizaciones, lo que no permite comparar los resultados. Sin embargo, la sobrevida a cinco años luego de la resección de metástasis pulmonares ha sido de $43 \%$ a $53 \%$ en sarcomas uterinos ${ }^{8,19}$, de $27 \%$ a $50,6 \%$ en leiomiosarcomas $^{18,31,36}$, y de $45 \%$ en los sarcomas estromales $^{36}$. Lo que sugiere que en pacientes seleccionados la resección quirúrgica es una excelente alternativa terapéutica, con resultados similares a la resección de metástasis pulmonares en otros tumores primarios.

Los factores pronósticos de la resección de metástasis pulmonares han sido extensamente estudiados. En los cánceres cérvico-uterinos, menos de 2 metástasis e histología escamosa se han asociado a un mayor intervalo libre de enferme$\operatorname{dad}^{26,31}$. Y en sarcomas uterinos la presencia de metástasis unilaterales se asoció a un mejor

\section{REFERENCIAS}

1. Kronlein RU. Ueber Lungenchirurgie. Berl Klin Wochnschr 1884; 21: 129-32.

2. WeinleChNer JW. Zur Kasuistik der Turmoren an der Brustwand und deren Behandlung (Resektion pronóstico y una resección incompleta fue factor de mal pronóstico ${ }^{19,32}$. En general, en los cánceres ginecológicos los factores de mal pronóstico identificados son: un intervalo libre de enfermedad menor de 12 ó 24 meses, mayor número de metástasis pulmonares, metástasis de mayor tamaño y un cáncer primario cérvico-uterino ${ }^{31,36}$.

La mayoría de las metástasis pulmonares se detectan en exámenes de seguimiento una vez controlado el tumor primario ${ }^{5}$. Esta situación también se repite en los tumores ginecológicos, pese a que su búsqueda sistemática se realiza con menor frecuencia. Sólo $41 \%$ a $77 \%$ de los pacientes con metástasis de cánceres ginecológicos tiene síntomas al momento del diagnóstico ${ }^{28,36}$. Debido a los beneficios en sobrevida con la resección de metástasis pulmonares, sería recomendable realizar estudios dirigidos (radiografía de tórax 0 idealmente TAC de tórax) para la detección de metástasis pulmonares durante el seguimiento de estas pacientes. Ante la presencia de un nódulo pulmonar único, debe siempre considerarse la posibilidad de un cáncer pulmonar primario, especialmente en pacientes con antecedentes de tabaquismo, debiendo realizarse un estudio apropiado.

En síntesis, la resección de metástasis pulmonares de tumores de origen ginecológico es un procedimiento aceptado, seguro y que prolonga la sobrevida en pacientes seleccionadas. Los criterios generales para la resección de metástasis pulmonares, utilizados en otros tumores primarios, deberían emplearse para determinar las pacientes que se pueden beneficiar de este procedimiento. Al igual que en otros tipos de cáncer, la resección completa de las metástasis, un intervalo libre de enfermedad prolongado y un menor número de metástasis son los factores pronósticos más importantes. En el seguimiento de las pacientes operadas de tumores ginecológicos debe incluirse exámenes destinados a la detección de metástasis pulmonares.

der Rippen, Eroffnung der Brusthohle, partielle Entfernung der Lunge). Wien Med Wochenschr 1882; 32: 589-91, 624-8.

3. BARNEY JD, ChURCHIL CE. Adenocarcinoma of the kidney with metastasis to the lung. J Urol 1939; 42: 269-76. 
4. Martini N, McCormack PM. Evolution of the surgical management of pulmonary metastases. Chest Surg Clin North Am 1998; 8: 13-27.

5. Pastorino U, Grunenwaid D. Pulmonary Metastasis. In Thoracic Surgery, $2^{\text {nd }}$ edition; Pearson FG, Cooper JD, Deslauriers J, Ginsberg RS, Hiebert CA, Patterson GA and Urschel HC eds. Churchill Livingstone, Philadelphia, Pennsylvania, 2002.

6. McCormack P. Surgical resection of pulmonary metastases. Semin Surg Oncol 1990; 6: 297-302.

7. Pastorino U, Buyse M, Friedel G, Ginsberg RJ, Girard P, Goldstraw P et al. Long term results of lung metastasectomy: prognostic analyses based on 5206 cases. J Thorac Cardiovasc Surg 1997; 113: $37-49$

8. Mountain CF, McMurtrey MJ, Hermes KE. Surgery for pulmonary metastases: A 20-year experience. Ann Thorac Surg 1984; 38: 323-30.

9. Monteiro A, Arce N, Bernardo J, Eugénio L, ANTUNES MJ. Surgical resection of lung metastases from epithelial tumors. Ann Thorac Surg 2004; 77: 431-7.

10. Beattie ej, Harvey JC, Marcove R, Martini N. Results of multiple pulmonary resections for metastatic osteogenic sarcoma after two decades. J Surg Oncol 1991; 46: 154-5.

11. Casson AG, Putnam JB, Natarajan G, Johnston DA, Mountain C, McMurtRey M, Roth JA. Five-year survival after pulmonary metastasectomy for adult soft tissue sarcoma. Cancer 1992; 69: 662-8.

12. McCormack PM, BuRt ME, Bains MS, Martini N, Rusch VW, GinsBerg RJ. Lung resection for colorectal metastases. 10-year results. Arch Surg 1992; 127: 1403-6.

13. McAfee MK, Aluen MS, Trastek VF, Ilstrup DM, Deschamps C, Pairolero PC. Colorectal lung metastases. Ann Thorac Surg 1992; 53: 780-5.

14. Headrick JR, Muler DL, Nagorney DM, Auen MS, Deschamps C, Trastek V, Pairolero PC. Surgical treatment of hepatic and pulmonary metastases from colon cancer. Ann Thorac Surg 2001; 71: 975-80.

15. Murthy SC, Kim K, Rice TW, Rajeswaran J, Bukowski $\mathrm{R}$, DeCamp M, Blackstone EH. Can we predict long-term survival after pulmonary metastasectomy for renal cell carcinoma? Ann Thorac Surg 2005; 79: 996-1003.

16. Cerfolo RJ, Aluen MS, Deschamps C, Daly RC, Walrichs St, Trastek V, Pairolero PC. Pulmonary resection of metastatic renal cell carcinoma. Ann Thorac Surg 1994; 57: 339-44.
17. Kavoluus JP, Mastorakos DP, Pavlovich C, Russo P, BURT ME, BRADY MS. Resection of metastatic renal cell carcinoma. J Clin Oncol 1998; 16: 2261-6.

18. McCormack PM, Martini N. The changing role of surgery for pulmonary metastases. Ann Thorac Surg 1979; 28: 139-45.

19. Levenback C, Rubin S, McCormack PM, Hoskins WJ, AtKinson EN, LeWIS JL. Resection of Pulmonary Metastases from Uterine Sarcomas. Gynecologic Oncology 1992; 45: 202-5.

20. Piltz S, Meimarakis G, Wichmann M, Hatz R, SCHILDBERG FW, FueRST H. Long-term results after pulmonary resection of renal cell carcinoma metastases. Ann Thorac Surg 2002; 73: 1082-7.

21. Ferson PF, KeEnan RJ, LuKetich JD. The role of VideoAssisted Thoracic Surgery in Pulmonary Metastases. Chest Surg Clin North Am 1998; 8: 59-76.

22. McCormack PM, GinsBerg KB, Bains MS, Burt ME, MARTIN N, Rusch VW, GinsBerg RJ. Accuracy of lung imaging in metastases with implications for the role of thoracoscopy. Ann Thorac Surg 1993; 56: 863-6.

23. McCormac PM Bains MS, Begg CB, Burt ME, Downey RJ, PANICEK DM ET aL. Role of videoassisted thorcic surgery in the treatment of pulmonary metastases: Results of a prospective trial. Ann Thorac Surg 1996; 62: 213-17.

24. Jemal A, Murray T, Ward E. Cancer statistics, 2005. CA Cancer J Clin 2005; 55(1): 10-30. Erratum in: CA Cancer J Clin 2005; 55(4): 259.

25. Anuario de estadísticas vitales año 2003, Institituto Nacional de Estadísticas, 2005. http:// www.ine.cl/ine/canales/chile_estadistico/ demografia_y_vitales/estadisticas_vitales/ estadisticas_vitales.php

26. Yamamoto K, Hiroyuki Y, Shiromizu K, Saito T, Kuzuya K, Tsunemasu R, Kamura T. Pulmonary Metastasectomy for Uterine Cervical Cancer: A Multivariate Analysis. Ann Thorac Surg 2004; 77: 1179-82.

27. Barter JF, Soong SJ, Hatch KD, OrR JW, Shingleton HM. Diagnosis and Treatment of Pulmonary Metastases from Cervical Carcinoma. Gynecologic Oncology 1990; 38: 347-51.

28. Imachi M, Tsukamoto N, Matsuyama T, Nakano $\mathrm{H}$. Pulmonary Metastasis from Carcinoma of the Uterine Cervix. Gynecologic Oncology 1989; 33: 189-92.

29. Aubry MC, Myers JL, Colby TV, Leslie KO, TazelaAr HD. Endometrial stromal sarcoma metastatic to the lung. A detailed analysis of 16 patients. Am J Surg Pathol 2002; 26: 440-9. 
30. TOREK F. Removal of metastatic carcinoma of the lung and mediastinum: suggestions as to technique. Arch Surg 1930; 21: 1416-24.

31. Anraku M, Yokoi K, Nakagawa K, Fujisawa $T$, Nakajima J, AkiYama H et al. Pulmonary metastases from uterine malignancies: Results of surgical resection in 133 patients. J Thorac Cardiovasc Surg 2004; 127: 1107-12.

32. Otzuka I, Ono I, Akamatsu H, Sunamori M, Aso T. Pulmonary metastasis from endometrial carcinoma. Int J Gynecol Cancer 2002; 12: 208-13.

33. Fuler AF, Scanell JG, Wilkins EW. Pulmonary resection for metastases from gynecological cancers: MGH experience. Gynecol Oncol 1985; 22: 174-80.

34. Tews CJ, Beecher CR. Pulmonary metastasis of carcinoma of the cervix: a retrospective study. Cancer 1982; 49: 1705-9.

35. Seki M, Nakagawa K, Tsuchiya S, Matsubara T, KINOSHITA I, Weng SY ET AL. Surgical treatment of pulmonary metastases from uterine cervical cancer. Operation method by lung tumor size. J Thorac Cardiovasc Surg 1992; 104: 876-81.

36. Clavero JM, Deschamps C, Cassivi SD, Auen MS, Nichols FC, BarRetTe BA et aL. Gynecologic Cancers: Factors Affecting Survival After Pulmonary Metastasectomy. Ann Thorac Surg 2006; 81: 2004-7.

37. Bouros D, Papadakis K, Siafakas N, Fuluer A. Natural history of patients with pulmonary metastases from uterine cancer. Cancer 1996; 78: 441-7.

38. Forney JP, Buschbaum HJ. Classifying, staging, and treating uterine sarcomas. Contemp Ob Gyn 1981; 18(3): 47, 50, 55-56, 61-62, 64, 69.
39. Major FJ, Biessing JA, Silverberg SG et al. Prognostic factors in early-stage uterine sarcoma. A Gynecologic Oncology Group study. Cancer 1993; 71 (4 Suppl): 1702-9.

40. Omura GA, Blessing JA, Major F et al. A randomized clinical trial of adjuvant adriamycin in uterine sarcomas: a Gynecologic Oncology Group Study. J Clin Oncol 1985; 3: 1240-5.

41. Kohorn EI, Schwartz PE, Chambers JT ET aL. Adjuvant therapy in mixed mullerian tumors of the uterus. Gynecol Oncol 1986; 23: 212-21.

42. Sutton GP, Biessing JA, Rosenshein N et al. Phase II trial of ifosfamide and mesna in mixed mesodermal tumors of the uterus (a Gynecologic Oncology Group study). Am J Obstet Gynecol 1989; 161: 309-12.

43. Sutton G, Blessing JA, Park R et al. Ifosfamide treatment of recurrent or metastatic endometrial stromal sarcomas previously unexposed to chemotherapy: a study of the Gynecologic Oncology Group. Obstet Gynecol 1996; 87 (5 Pt 1): 747-50.

44. Sutton GP, Blessing JA, Barrett RJ et al. Phase II trial of ifosfamide and mesna in leiomyosarcoma of the uterus: a Gynecologic Oncology Group study. Am J Obstet Gynecol 1992; 166: 556-9.

45. Sutton G, Brunetto VL, KiLgore L et al. A phase III trial of ifosfamide with or without cisplatin in carcinosarcoma of the uterus: A Gynecologic Oncology Group Study. Gynecol Oncol 2000; 79: 147-53.

46. Berchum A, Rubin SC, Hoskins WJ, Saigo PE, Pierce VK, LEWIA JL JR. Treatment of uterine leiomyosarcoma. Obstet Gynecol 1988; 71: 845-50. 